\title{
Europäischer Tarifbericht des WSI - 2012/2013
}

Seit Ausbruch der Weltwirtschaftskrise 2009ff. hat sich innerhalb Europas ein neues lohnpolitisches Entwicklungsmuster herausgebildet, das in den meisten europäischen Staaten durch anhaltende Reallohnverluste geprägt ist. Auch die aktuelle Lohnentwicklung der Jahre 2012 und 2013 fügt sich nahtlos in dieses Schema ein. Allein im Jahr 2012 blieben die Nominallohnzuwächse in 20 von 28 EU-Staaten hinter der Preissteigerungsrate zurück. Für 2013 wird die Bilanz nur deshalb leicht besser aussehen, weil die Inflationsraten einen deutlich rückläufigen Trend aufweisen. Der durch die europäische Austeritätspolitik verursachte Einbruch in der gesamtwirtschaftlichen Nachfrage wird durch die anhaltend schwache Lohnentwicklung weiter verschärft. Die Lohnpolitik trägt damit ihrerseits dazu bei, dass sich die ökonomische Stagnation in Europa weiter fortsetzen wird.

THORSTEN SCHULTEN

\section{1. Ökonomische Rahmenbedingungen der Tarifpolitik}

\subsection{Allgemeine Wirtschaftsentwicklung}

In den meisten europäischen Ländern stehen die Tarifauseinandersetzungen nach wie vor unter dem Vorzeichen von ökonomischer Stagnation und Krise. Nachdem sich die Konjunktur bereits 2011 merklich abgekühlt hat, ist die Europäische Union (EU 28) als Ganzes 2012 in eine offene Rezession geschlittert, die auch im Jahr 2013 weiter anhalten wird (vgl. Europäische Kommission 2013a; Horn et al. 2013). Im EU-Durchschnitt ging das Bruttoinlandsprodukt (BIP) 2012 um 0,4\% zurück. Im Vorjahr lag das EU-weite Wirtschaftswachstum demgegenüber noch bei 1,5\%. Für 2013 erwartet die Europäische Kommission, dass die ökonomische Stagnation mit einem Rückgang des BIP von $0,1 \%$ weiter anhält (Tabelle 1$).{ }^{1}$

Zwischen den einzelnen EU-Staaten zeigt sich ein deutlich gespaltener Konjunkturverlauf. 2012 befanden sich knapp die Hälfte aller EU-Staaten (13 von 28) in einer Rezession, wobei der Wachstumseinbruch in den südeuropäischen Ländern sowie einigen osteuropäischen Staaten am stärksten ausfiel. Das Schlusslicht bildete Griechenland mit einem BIP-Rückgang von $6,4 \%$, gefolgt von Portugal mit minus 3,2 \%, Italien und Zypern mit Werten von minus 2,4 und Slowenien von $2,3 \%$. Von den 15 EU-Staaten mit po- sitiven Wachstumsraten lag der BIP-Zuwachs in zehn Ländern unterhalb von $1 \%$. Hierzu gehörte neben den meisten westeuropäischen Staaten auch Deutschland, das lediglich ein BIP-Wachstum von 0,7 \% realisieren konnte. Die höchsten Wachstumsraten konnten wie bereits 2011 die baltischen Staaten verzeichnen, wobei Lettland mit einem BIP-Zuwachs von 5,6\% den Spitzenplatz einnahm.

Nach den Prognosen der Europäischen Kommission wird sich auch im Jahr 2013 in vielen Ländern die ökonomische Stagnation weiter fortsetzen. Insbesondere in Südeuropa ist kein Ende der Krise abzusehen. Besonders dramatisch ist die Situation in Zypern, wo ein Rückgang des BIP von 8,7 \% erwartet wird. Auch in Griechenland soll die Entwicklung des BIP erneut um mehr als $4 \%$ zurückgehen und wird damit im sechsten Jahr in Folge negativ sein. Die Krise in Südeuropa wirkt sich auch weiterhin dämpfend auf die Konjunkturentwicklung in den exportorientierten Ländern Nordund Westeuropas aus. Mit Ausnahme von Irland und Schweden wird keines dieser Länder ein BIP-Wachstum oberhalb

\footnotetext{
1 Im Folgenden wird, falls nicht anders ausgewiesen, auf Daten der Annual Macro-Economic Database (AMECO) zurückgegriffen, die von der Generaldirektion Wirtschaft und Finanzen (GD ECFIN) der Europäischen Kommission bereitgestellt wird (http://ec.europa.eu/economy_finance/db_ indicators/ameco/index_en.htm). Bei den Angaben für 2013 handelt es sich um Prognosedaten der Europäischen Kommission (vgl. Europäische Kommission 2013a).
} 
der $1 \%$-Marke aufweisen können. Lediglich die baltischen Staaten werden ein recht kräftiges Wachstum von $3 \%$ und mehr erreichen. In Deutschland wird der BIP-Zuwachs 2013 mit prognostizierten $0,4 \%$ noch einmal unterhalb der ebenfalls schon schwachen Entwicklung des Vorjahres liegen.

\subsection{Die Lage auf dem Arbeitsmarkt}

Neben der allgemeinen konjunkturellen Entwicklung bildet die Lage auf dem Arbeitsmarkt einen weiteren zentralen Faktor für die Tarifauseinandersetzungen, die sich direkt auf die Verhandlungs- und Durchsetzungsmacht der Tarifvertragsparteien auswirkt. In vielen europäischen Ländern wird derzeit die Position der Gewerkschaften durch eine anhaltend hohe Arbeitslosigkeit geschwächt. Im Jahresdurchschnitt 2012 waren EU-weit mehr als 25,5 Mio. Menschen offiziell arbeitslos, was einer Arbeitslosenquote von 10,5\% entspricht (Tabelle 1). Für 2013 wird angesichts der anhaltenden ökonomischen Stagnation ein weiterer Anstieg der Arbeitslosigkeit auf 11,1\% erwartet.

Spiegelbildlich zur Konjunkturlage bestehen auch bei dem Ausmaß der Arbeitslosigkeit zwischen den einzelnen EU-Staaten sehr große Unterschiede. Die höchsten Arbeitslosenquoten existierten 2012 in Griechenland und Spanien, wo jeweils ein Viertel aller Beschäftigten erwerbslos war. Weitere 13 EU-Staaten wiesen Arbeitslosenquoten von $10 \%$ und mehr auf. Hierzu gehörten in Westeuropa Frankreich und Irland, die südeuropäischen Länder Italien, Portugal und Zypern sowie zahlreiche osteuropäische Staaten. Letztere umfassen auch die baltischen Staaten, in denen trotz überdurchschnittlich hoher Wachstumsraten die Arbeitslosigkeit nur sehr langsam zurückgeht. Auf der anderen Seite gab es vier Länder mit Arbeitslosenquoten von unter $6 \%$, darunter Deutschland mit 5,5\%, die Niederlande mit 5,3\%, Luxemburg mit 5,1 \% und Österreich mit 4,3\%. Der für die gesamte EU prognostizierte weitere Anstieg der Arbeitslosenquoten im Jahr 2013 geht vor allem auf das Konto der süd- und osteuropäischen Staaten, während sich die Arbeitslosenquoten in Nord- und Westeuropa zumeist nur unwesentlich verändern.

\subsection{Preis- und Produktivitätsentwicklung}

Neben der Konjunkturentwicklung und der Lage auf dem Arbeitsmarkt ist die Entwicklung der Preise und der Arbeitsproduktivität ein wichtiger Orientierungspunkt für die Tarifauseinandersetzungen in Europa (Tabelle 2). Die Summe beider Indikatoren bildet den lohnpolitischen Verteilungsspielraum, dessen Ausschöpfung durch entsprechende Lohnerhöhungen den Beschäftigten eine gleichgewichtige Partizipation an der allgemeinen Wirtschaftentwicklung garantiert und das Verhältnis zwischen Arbeits- und Kapitaleinkommen konstant hält. Bei der Definition des lohnpolitischen Verteilungsspielraumes als Summe aus Preis- und Produktivitätsentwicklung ist in der Wirtschaftswissenschaft strittig, welche konkreten Indikatoren insbesondere
TABELLE 1

Wachstum und Arbeitslosigkeit in der Europäischen Union 2010-2013* Angaben in Prozent

\begin{tabular}{|c|c|c|c|c|c|c|c|c|}
\hline & \multicolumn{4}{|c|}{ Bruttoinlandsprodukt } & \multicolumn{4}{|c|}{ Arbeitslosigkeit } \\
\hline & 2010 & 2011 & 2012 & 2013 & 2010 & 2011 & 2012 & 2013 \\
\hline \multicolumn{9}{|l|}{ Nordeuropa } \\
\hline Dänemark & 1,6 & 1,1 & $-0,5$ & 0,7 & 7,5 & 7,6 & 7,5 & 7,7 \\
\hline Finnland & 3,3 & 2,8 & $-0,2$ & 0,3 & 8,4 & 7,8 & 7,7 & 8,1 \\
\hline Schweden & 6,6 & 3,7 & 0,8 & 1,5 & 8,6 & 7,8 & 8,0 & 8,3 \\
\hline \multicolumn{9}{|l|}{ Westeuropa } \\
\hline Belgien & 2,4 & 1,8 & $-0,2$ & 0,0 & 8,3 & 7,2 & 7,6 & 8,0 \\
\hline Deutschland & 4,2 & 3,0 & 0,7 & 0,4 & 7,1 & 5,9 & 5,5 & 5,4 \\
\hline Frankreich & 1,7 & 1,7 & $-0,0$ & $-0,1$ & 9,7 & 9,6 & 10,2 & 10,6 \\
\hline Großbritannien & 1,8 & 1,0 & 0,3 & 0,6 & 7,8 & 8,0 & 7,9 & 8,0 \\
\hline Irland & $-0,8$ & 1,4 & 0,9 & 1,1 & 13,9 & 14,7 & 14,7 & 14,2 \\
\hline Luxemburg & 2,9 & 1,7 & 0,3 & 0,8 & 4,6 & 4,8 & 5,1 & 5,5 \\
\hline Niederlande & 1,6 & 1,0 & $-1,0$ & $-0,8$ & 4,5 & 4,4 & 5,3 & 6,9 \\
\hline Österreich & 2,1 & 2,7 & 0,8 & 0,6 & 4,4 & 4,2 & 4,3 & 4,7 \\
\hline \multicolumn{9}{|l|}{ Südeuropa } \\
\hline Griechenland & $-4,9$ & $-7,1$ & $-6,4$ & $-4,2$ & 12,6 & 17,7 & 24,3 & 27,0 \\
\hline Italien & 1,7 & 0,4 & $-2,4$ & $-1,3$ & 8,4 & 8,4 & 10,7 & 11,8 \\
\hline Malta & 2,9 & 1,7 & 0,8 & 1,4 & 6,9 & 6,5 & 6,4 & 6,3 \\
\hline Portugal & 1,9 & $-1,6$ & $-3,2$ & $-2,3$ & 12,0 & 12,9 & 15,9 & 18,2 \\
\hline Spanien & $-0,3$ & 0,4 & $-1,4$ & $-1,5$ & 20,1 & 21,7 & 25,0 & 27,0 \\
\hline Zypern & 1,3 & 0,5 & $-2,4$ & $-8,7$ & 6,3 & 7,9 & 11,9 & 15,5 \\
\hline \multicolumn{9}{|l|}{ Osteuropa } \\
\hline Bulgarien & 0,4 & 1,8 & 0,8 & 0,9 & 10,3 & 11,3 & 12,3 & 12,5 \\
\hline Estland & 3,3 & 8,3 & 3,2 & 3,0 & 16,9 & 12,5 & 10,2 & 9,7 \\
\hline Kroatien & $-2,3$ & 0,0 & $-2,0$ & & 11,8 & 13,5 & 15,9 & 19,1 \\
\hline Lettland & $-0,9$ & 5,5 & 5,6 & 3,8 & 19,8 & 16,2 & 14,9 & 13,7 \\
\hline Litauen & 1,5 & 5,9 & 3,6 & 3,1 & 18,0 & 15,3 & 13,3 & 11,8 \\
\hline Polen & 3,9 & 4,5 & 1,9 & 1,1 & 9,7 & 9,7 & 10,1 & 10,9 \\
\hline Rumänien & $-1,1$ & 2,2 & 0,7 & 1,6 & 7,3 & 7,4 & 7,0 & 6,9 \\
\hline Slowakei & 4,4 & 3,2 & 2,0 & 1,0 & 14,5 & 13,6 & 14,0 & 14,5 \\
\hline Slowenien & 1,2 & 0,6 & $-2,3$ & $-2,0$ & 7,3 & 8,2 & 8,9 & 10,0 \\
\hline Ungarn & 1,3 & 1,6 & $-1,7$ & 0,2 & 11,2 & 10,9 & 10,9 & 11,4 \\
\hline Tschechien & 2,5 & 1,9 & $-1,3$ & $-0,4$ & 7,3 & 6,7 & 7,0 & 7,5 \\
\hline Gesamte EU 28 & 2,1 & 1,5 & $-0,4$ & $-0,1$ & 9,7 & 9,7 & 10,5 & 11,1 \\
\hline
\end{tabular}

*Bruttoinlandsprodukt = Veränderung des BIP in \% gegenüber dem Vorjahr. Arbeitslosenquote $=$ Anzahl der Arbeitslosen in \% der zivilen Erwerbsbevölkerung (Eurostat-Definition). Angaben für 2013: Prognose der Europäischen Kommission.

Quelle: AMECO Datenbank, Version: 3.5.2013.

für die Preisentwicklung verwendet werden sollen. Gegenüber möglichen alternativen Indikatoren (wie z. B. der Kerninflationsrate, der Zielinflationsrate der Europäischen Zentralbank (EZB) oder der Entwicklung der Erzeugerpreise) wird im Folgenden bei der Bestimmung der nationalen Verteilungsspielräume die jeweils nationale Entwicklung der Verbraucherpreise zugrunde gelegt, wobei für den internationalen Vergleich der Harmonisierte Verbraucherpreisindex (HVPI) als Grundlage verwendet wird. Aus Ar- 
TABELLE 2

Preise, Arbeitsproduktivität und Verteilungsspielraum in der Europäischen Union 2012-2013*

Angaben in Prozent

\begin{tabular}{|c|c|c|c|c|c|c|c|c|c|c|c|c|}
\hline & \multicolumn{4}{|c|}{ Preise } & \multicolumn{4}{|c|}{ Arbeitsproduktivität } & \multicolumn{4}{|c|}{ Verteilungsspielraum } \\
\hline & 2010 & 2011 & 2012 & 2013 & 2010 & 2011 & 2012 & 2013 & 2010 & 2011 & 2012 & 2013 \\
\hline \multicolumn{13}{|l|}{ Nordeuropa } \\
\hline Dänemark & 2,2 & 2,7 & 2,4 & 1,1 & 3,9 & 1,5 & $-0,0$ & 0,9 & 6,1 & 4,2 & 2,4 & 2,0 \\
\hline Finnland & 1,7 & 3,3 & 3,2 & 2,4 & 3,4 & 1,7 & $-0,5$ & 0,7 & 5,1 & 5,0 & 2,7 & 3,1 \\
\hline Schweden & 1,9 & 1,4 & 0,9 & 0,9 & 5,5 & 1,4 & 0,1 & 1,4 & 7,4 & 2,7 & 1,1 & 2,3 \\
\hline \multicolumn{13}{|l|}{ Westeuropa } \\
\hline Belgien & 2,3 & 3,4 & 2,6 & 1,3 & 1,7 & 0,4 & $-0,4$ & $-0,1$ & 4,1 & 3,8 & 2,3 & 1,2 \\
\hline Deutschland & 1,2 & 2,5 & 2,1 & 1,8 & 3,6 & 1,6 & $-0,4$ & $-0,1$ & 4,7 & 4,1 & 1,7 & 1,6 \\
\hline Frankreich & 1,7 & 2,3 & 2,2 & 1,2 & 1,9 & 1,2 & 0,1 & $-0,1$ & 3,7 & 3,5 & 2,4 & 1,1 \\
\hline Großbritannien & 3,3 & 4,5 & 2,8 & 2,8 & 1,6 & 0,5 & $-0,9$ & 0,0 & 4,9 & 5,0 & 1,9 & 2,8 \\
\hline Irland & $-1,6$ & 1,2 & 1,9 & 1,3 & 3,4 & 3,3 & 1,5 & 1,0 & 1,9 & 4,4 & 3,5 & 2,3 \\
\hline Luxemburg & 2,8 & 3,7 & 2,9 & 1,9 & 1,1 & $-1,2$ & $-1,9$ & $-0,8$ & 3,9 & 2,5 & 1,0 & 1,1 \\
\hline Niederlande & 0,9 & 2,5 & 2,8 & 2,8 & 2,2 & 0,5 & $-0,1$ & 0,2 & 3,2 & 3,0 & 2,8 & 3,0 \\
\hline Österreich & 1,7 & 3,6 & 2,6 & 2,0 & 1,5 & 1,3 & $-0,3$ & $-0,1$ & 3,2 & 4,8 & 2,2 & 1,9 \\
\hline \multicolumn{13}{|l|}{ Südeuropa } \\
\hline Griechenland & 4,7 & 3,1 & 1,0 & $-0,8$ & $-2,4$ & $-1,6$ & 2,1 & $-0,7$ & 2,3 & 1,5 & 3,2 & $-1,5$ \\
\hline Italien & 1,6 & 2,9 & 3,3 & 1,6 & 2,8 & 0,2 & $-1,3$ & $-0,2$ & 4,5 & 3,1 & 2,0 & 1,4 \\
\hline Malta & 2,0 & 2,5 & 3,2 & 1,9 & 1,1 & $-1,0$ & $-1,2$ & $-0,4$ & 3,2 & 1,5 & 2,0 & 1,5 \\
\hline Portugal & 1,4 & 3,6 & 2,8 & 0,7 & 3,5 & $-0,0$ & 1,1 & 1,6 & 4,9 & 3,5 & 3,9 & 2,3 \\
\hline Spanien & 2,0 & 3,1 & 2,4 & 1,5 & 2,0 & 2,2 & 3,2 & 2,0 & 4,1 & 5,2 & 5,6 & 3,5 \\
\hline Zypern & 2,6 & 3,5 & 3,1 & 1,0 & 1,5 & 0,1 & 1,7 & $-2,2$ & 4,1 & 3,6 & 4,8 & $-1,2$ \\
\hline \multicolumn{13}{|l|}{ Osteuropa } \\
\hline Bulgarien & 3,0 & 3,4 & 2,4 & 2,0 & 5,3 & 5,4 & 5,4 & 3,1 & 8,4 & 8,8 & 7,7 & 5,1 \\
\hline Estland & 2,7 & 5,1 & 4,2 & 3,6 & 8,5 & 1,2 & 1,0 & 2,7 & 11,2 & 6,3 & 5,2 & 6,3 \\
\hline Kroatien & 1,1 & 2,2 & 3,4 & 3,1 & 3,0 & 2,4 & 2,0 & 3,3 & 4,1 & 4,6 & 5,4 & 6,4 \\
\hline Lettland & $-1,2$ & 4,2 & 2,3 & 1,4 & 4,0 & 14,8 & 2,9 & 1,9 & 2,8 & 19,1 & 5,2 & 3,3 \\
\hline Litauen & 1,2 & 4,1 & 3,2 & 2,1 & 7,0 & 3,8 & 1,8 & 1,8 & 8,2 & 7,9 & 5,0 & 3,9 \\
\hline Polen & 2,7 & 3,9 & 3,7 & 1,4 & 3,4 & 3,5 & 1,7 & 1,5 & 6,1 & 7,4 & 5,3 & 2,9 \\
\hline Rumänien & 6,1 & 5,8 & 3,4 & 4,3 & $-0,9$ & 3,3 & $-1,2$ & 1,1 & 5,2 & 9,1 & 2,2 & 5,5 \\
\hline Slowakei & 0,7 & 4,1 & 3,7 & 1,9 & 6,0 & 1,4 & 2,0 & 0,9 & 6,7 & 5,5 & 5,7 & 2,8 \\
\hline Slowenien & 2,1 & 2,1 & 2,8 & 2,2 & 3,5 & 2,2 & $-1,1$ & $-0,3$ & 5,6 & 4,3 & 1,7 & 1,8 \\
\hline Tschechien & 1,2 & 2,1 & 3,5 & 1,9 & 3,5 & 1,6 & $-1,7$ & $-0,4$ & 4,8 & 3,8 & 1,9 & 1,5 \\
\hline Ungarn & 4,7 & 3,9 & 5,7 & 2,6 & 0,6 & 1,2 & $-1,8$ & 0,2 & 5,3 & 5,2 & 3,9 & 2,8 \\
\hline EU 28 & 2,1 & 3,1 & 2,6 & 1,8 & 2,6 & 1,3 & 0,0 & 0,3 & 4,7 & 4,4 & 2,6 & 2,1 \\
\hline
\end{tabular}

*Preise $=$ Harmonisierter Verbraucherpreisindex (HVPI), Veränderung in \% gegenüber dem Vorjahr.

Arbeitsproduktivität $=$ Reales BIB pro Erwerbstatiger, Veränderung in \% gegenüber dem Vorjahr.

Verteilungsspielraum $=$ Summe aus Preis- und Arbeitsproduktivitätsentwicklung.

Angaben für 2013: Prognose der Europäischen Kommission.

Quelle: AMECO Datenbank der Europäischen Kommission, Version: 3.5.2013, Berechnungen des WSI.

beitnehmersicht stellen die Verbraucherpreise den wichtigsten ökonomischen Indikator dar, weil sie über das reale Lohnniveau und die damit verbundene Kaufkraft entscheiden. Dementsprechend bilden die nationalen Verbraucherpreise für die Gewerkschaften die bedeutsamste Orientierungsgröße bei den Tarifauseinandersetzungen.

Im Jahr 2012 stiegen die Verbraucherpreise im EUDurchschnitt um 2,6\% und damit etwas geringer als im Vorjahr mit 3,1\% (Tabelle 2). Angesicht der anhaltenden schwachen Konjunkturentwicklung erwartet die Europäische Kommission für 2013 eine deutlich schwächere Preisentwicklung von lediglich $1,8 \%$. Die Spannbreite bei den Inflationsraten bewegte sich 2012 zwischen 5,7 \% in Ungarn und $0,9 \%$ in Schweden. Bemerkenswert ist, dass auch die krisengeschüttelten Staaten Südeuropas 2012 trotz eines drastischen Einbruchs in der Binnennachfrage immer noch relativ hohe Preissteigerungen verzeichneten. Lediglich in Griechenland hat mittlerweile die Krise die Preisentwick- 
lung mit einer Inflationsrate von nur noch einem Prozent deutlich nach unten gedrückt. Nach den Vorhersagen der Europäischen Kommission wird 2013 in Griechenland als einzigem Land in der EU die Preisentwicklung mit minus $0,8 \%$ sogar negativ ausfallen.

Die Entwicklung der Arbeitsproduktivität ist vor dem Hintergrund der Rezession im Jahr 2012 EU-weit zum Erliegen gekommen, nachdem sich bereits im Vorjahr ein deutlich rückläufiger Trend andeutete (Tabelle 2). In insgesamt 13 EU-Staaten ging die Produktivität zurück, darunter am stärksten in Luxemburg (minus 1,9\%) sowie Ungarn (minus 1,8\%) und Tschechien (minus 1,7\%). Auch in Deutschland war die Produktivitätsentwicklung mit minus $0,4 \%$ leicht rückläufig. Signifikante Produktivitätszuwächse gab es hingegen in einigen osteuropäischen Ländern sowie in Griechenland und Spanien, wobei dies in den beiden letztgenannten Ländern vor allem auf den anhaltenden Beschäftigungsabbau zurückzuführen sein dürfte. Für 2013 prognostiziert die Europäische Kommission erneut eine äußerst schwache Produktivitätsentwicklung, die im EUDurchschnitt gerade mal bei einem Zuwachs von 0,3 \% liegt.

Da 2012 die Preisentwicklung wieder zurückgegangen und die Produktivität faktisch eingebrochen ist, hat sich rechnerisch der lohnpolitische Verteilungsspielraum im EUDurchschnitt mit 2,6 \% gegenüber 4,4 \% im Vorjahr deutlich verringert (Tabelle 2). Der größte Verteilungsspielraum existierte mit 7,7 \% in Bulgarien, gefolgt von einer Reihe weiterer osteuropäischer Länder mit Werten zwischen 5 und 5,7 \%. Diese Länder haben damit die ökonomischen Voraussetzungen geschaffen, den lohnpolitischen Aufholprozess gegenüber Westeuropa wieder aufzunehmen, der zuvor durch die Krise unterbrochen bzw. teilweise sogar rückgängig gemacht wurde. Lediglich in Slowenien und Tschechien lag der Verteilungsspielraum mit Werten von 1,7 bzw. 1,9\% unterhalb des EU-Durchschnittes.

Ein rechnerisch überdurchschnittlich hoher Verteilungsspielraum ergibt sich aufgrund der nach wie vor hohen Preis-und Produktivitätsentwicklung auch in einer Reihe südeuropäischer Staaten sowie in Irland. Deutschland weist hingegen mit 1,7 \% einen der niedrigsten Verteilungsspielräume in der EU auf, der lediglich noch von Luxemburg und Schweden unterboten wird. Angesichts der weiter rückläufigen Preisentwicklung wird sich der Verteilungsspielraum in der EU im Jahr 2013 mit nur noch 2,1 \% noch einmal verringern. Allerdings besteht zwischen den EUStaaten nach wie vor eine große Spannbreite, wobei erstmals seit dem Ausbruch der Krise 2009 der Verteilungsspielraum in zwei Ländern (Griechenland und Zypern) wieder negativ sein wird.

Während hier für die Berechnung des jeweils jahresbezogenen Verteilungsspielraumes auch die jahresbezogene Produktivität verwendet wird, wird für eine gesamtwirtschaftlich ausgerichtete Tarifpolitik in der Regel eine Orientierung an der Trendproduktivität empfohlen, die sich aus der durchschnittlichen Produktivitätsentwicklung mehrerer Jahre ergibt (vgl. z. B. Herr/Horn 2012). Eine solche mittelfristige Orientierung hat den Vorteil, dass sie zu einer Verstetigung der Lohnpolitik beitragen kann und prozyklische Effekte vermeidet, die sich z. B. dann ergeben können, wenn eine rückläufige Konjunkturentwicklung durch geringe Lohnzuwächse oder gar Lohnkürzungen weiter verstärkt wird. Legt man statt dem jeweils aktuellen Produktivitätszuwachs die mittelfristige Trendproduktivität zugrunde, die in der EU in den letzten zehn Jahren durchschnittlich bei $0,9 \%$ lag, so ergibt sich EU-weit für das Jahr 2012 ein Verteilungsspielraum von 3,5\% und für 2013 von $2,7 \%$.

\section{Die Entwicklung der Tariflöhne}

\subsection{Der EZB-Tariflohnindikator}

Die Analyse der Tariflohnentwicklung in Europa steht vor dem grundsätzlichen Problem, dass es bis heute keine offizielle europäische Tariflohnstatistik gibt. ${ }^{2}$ Die einzige offizielle Datenquelle auf europäischer Ebene bildet bislang der sogenannte "Indikator der Tarifverdienste“, der von der EZB als aggregierte Größe für die gesamte Euro-Zone berechnet wird. Demnach sind die Tariflöhne im Euroraum im Jahr 2012 nominal durchschnittlich um 2,1 \% angestiegen (Abbildung 1). Für das erste Halbjahr 2013 weisen die EZB-Daten eine Tariflohnerhöhung von 1,8 \% aus. Nach Abzug der Preissteigerungsrate ergibt sich danach für 2012 ein Rückgang der realen Tariflöhne um 0,4\%. Aufgrund der rückläufigen Preisentwicklung kommt es hingegen 2013 bei den Tariflöhnen zu einem minimalen Reallohnzuwachs von $0,2 \%$.

Seit Beginn der 2000er Jahre zeigt die Entwicklung des EZB-Tariflohnindikators sowohl nominal als auch real eine erstaunliche Konstanz. Während die nominale Tariflohnentwicklung im Wesentlichen zwischen 2 und 2,7\%

2 Seit einigen Jahren veröffentlicht das European Industrial Relations Observatory (EIRO) regelmäßig Daten über die Entwicklung der "Tariflöhne" in Europa. Nachdem die Berichte lange Zeit große methodische Mängel aufwiesen, die u. a. darin lagen, dass nicht immer klar zwischen Tarif- und Effektivlohndaten unterschieden wurde, wurde der Bericht seit dem Jahr 2011 erstmals auf eine neue verbesserte Datengrundlage gestellt. Hierbei wird ähnlich wie in dem vorliegenden Bericht auf die wenigen verfügbaren nationalen Datenquellen überTariflöhne zurückgegriffen (vgl. für den aktuellsten EIRO-Report: Aumayr-Pintar/Fric 2013). Qualitative Berichte über dieTarifauseinandersetzungen in den einzelnen EU-Staaten finden sich in den laufenden nationalen Beiträgen zum EIRO (http://www.eurofound.europa.eu/ eiro) sowie in dem gemeinsam vom Europäischen Gewerk schaftsinstitut und dem Amsterdam Institute for Advanced Labour Studies (AIAS) herausgegebenen Collective Bargaining Newsletter (http://www.etui.org/E-Newsletters/ Collective-bargaining-newsletter). 
ABB. 1

\section{Entwicklung des EZB-Indikators der Tarifverdienste für die Euro-Zone 2000 - 2013*}

Veränderungen in Prozent zum Vorjahr Nominal $\quad$ Real

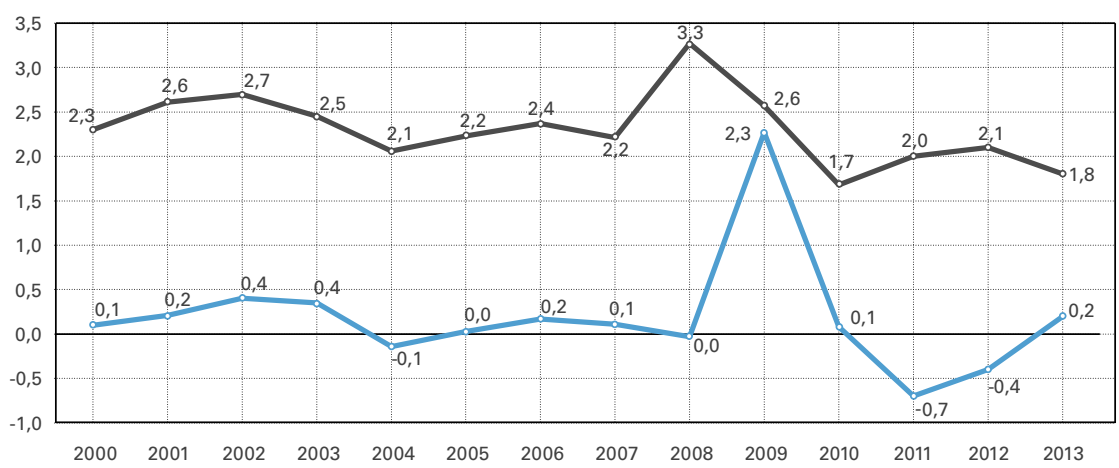

*reale Entwicklung deflationiert um den Harmonisierten Verbraucherindex (HVPI); Daten für 2013 beziehen sich auf das erste Halbjahr.

Quelle: Europäische Zentralbank (http://sdw.ecb.europa.eu/browse.do?node=2120788) Berechnungen des WSI.

\section{ABB. 2}

\section{Nominale Entwicklung der Tariflöhne 2012 - 2013*}

Veränderungen in Prozent zum Vorjahr

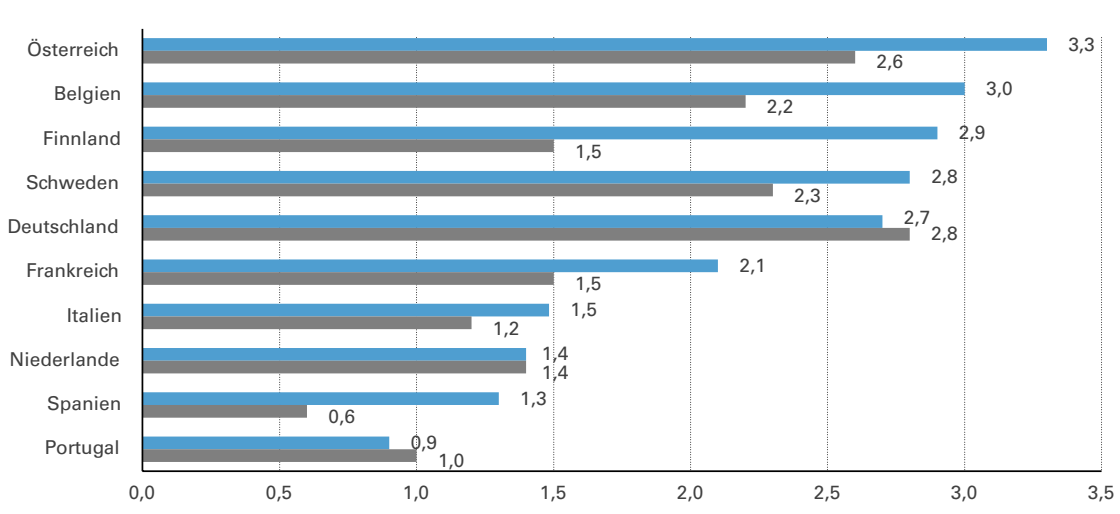

*2013: vorläufige Daten auf der Grundlage des ersten Halbjahres.

Quelle: Nationale Angaben; Berechnungen des WSI. schwankt, liegt sie real zumeist wenige Zehntel-Prozent oberhalb der Inflationsrate. Eine Ausnahme bildeten lediglich das Jahr 2008, wo es vor dem Hintergrund steigender Preise auch zu einem ungewöhnlich starken nominalen Tariflohnanstieg kam, sowie das Krisenjahr 2009, in dem der Preisanstieg extrem niedrig war und damit erstmals zu einem kräftigen Anstieg der realen Tarifverdienste führte.

Bei dem EZB-Tariflohnindikator handelt es sich um sogenannte „experimentelle Daten“, die durch die Zusammenführung nicht harmonisierter nationaler Datenquellen berechnet werden und deshalb lediglich als Näherungswert an die Wirklichkeit angesehen werden können (Schubert 2012). Bei der Berechnung des EZB-Indikators werden Tariflohndaten aus insgesamt zehn Ländern berücksichtigt, die insgesamt $98 \%$ der Euro-Zone abdecken. ${ }^{3}$ Allerdings werden von der EZB weder die verwendeten nationalen Daten veröffentlicht, noch werden die zugrunde liegenden nationalen Datenquellen öffentlich preisgegeben, sodass der EZB-Tariflohnindikator insgesamt sehr intransparent und kaum nachprüfbar ist.

\subsection{Die Tariflohnentwicklung in ausgewählten EU-Staaten}

Mangels einer originär europäischen Datenquelle muss bei der Analyse der Tariflohnentwicklung in Europa notgedrungen auf nicht harmonisierte nationale Datensätze zurückgegriffen werden, die in den meisten Fällen entweder von den nationalen Statistikämtern oder von den nationalen Arbeitsministerien erhoben werden und nur für eine begrenzte Anzahl von Ländern verfügbar sind. ${ }^{4}$ Die Analyse der zehn hier berücksichtigten EU-Staaten zeigt insgesamt ein recht uneinheitliches Bild, was darauf hindeutet, dass die Tarifpolitik deutlich von den jeweiligen ökonomischen Rahmenbedingungen geprägt wird. Im Jahr 2012 lag die Spannbreite der Tariflohnzuwächse zwischen 3,3 \% in Österreich und 0,9\% in Portugal. Deutschland befand sich mit einer Tariflohnsteigerung von 2,7 \% im oberen Mittelfeld (Abbildung 2).

Generell fallen die Tariflohnzuwächse in Ländern, die sich in einer offenen Rezession befinden, deutlich schwächer aus als in Ländern, die nach wie vor positives Wachstum aufweisen. Allerdings reagieren die Tariflöhne aufgrund mehrjähriger Tarifvereinbarungen oft erst mit einer bestimmten Zeitverzögerung auf die konjunkturelle Entwick-
3 Bei den Ländern, die in dem EZB-Tariflohnindikator berücksichtigt werden, handelt es sich um Belgien, Deutschland, Finnland, Frankreich, Italien, die Niederlande, Österreich, Portugal, Slowenien und Spanien (Schubert 2012). Für Deutschland werden die Tariflohndaten der Deutschen Bundesbank genutzt, die gegenüber den im Folgenden in diesem Bericht verwendeten Daten des WSI-Tarifarchivs die Tariflohndynamik etwas unterzeichnen. Zu den unterschiedlichen Tariflohnstatistiken in Deutschland vgl. Bispinck/Schulten (2012).
4 Im Rahmen eines von der Europäischen Kommission geförderten internationalen Forschungsprojektes über "Collectively Agreed Wages in Europe" (CAWIE), an dem das WSI als deutscher Partner und Ko-Koordinator beteiligt war, wurden die nationalen Datenquellen über Tariflöhne im Hinblick auf ihre Vergleichbarkeit und Qualität analysiert und Möglichkeiten zum Aufbau einer europäischen Tariflohndatenbank geprüft (Van Gyes 2012). Für mehr Informationen über die Ergebnisse des CAWIE-Projekt vgl. http://hiva.kuleuven.be/nl/extra/CAWIE.php. 
lung. So hat in vielen Ländern die verschlechterte Konjunkturlage im Jahr 2012 dazu beigetragen, dass die Tariflöhne im Jahr 2013 deutlich langsamer ansteigen. Auf der Grundlage der Daten für das erste Halbjahr 2013 lässt sich dies für sieben der hier berücksichtigten Länder bestätigen. Zu den Ausnahmen gehört vor allem Deutschland, wo 2013 die Tariflohnsteigerung mit 2,8 \% die Entwicklung des Vorjahres sogar leicht übertrifft. Sollten sich die Daten so für das gesamte Jahr 2013 bestätigen, so würde Deutschland sogar erstmals seit Langem den Spitzenplatz bei den Tariflohnerhöhungen einnehmen. Demgegenüber liegen die Tariflohnzuwächse in sechs Staaten bei 1,5\% und weniger, wobei Spanien mit einem Zuwachs von lediglich 0,6 \% das Schlusslicht bildet.

Nach Abzug der Inflationsrate gingen die Tariflöhne 2012 in sechs von zehn Ländern zurück (Abbildung 3). Die höchsten Reallohnverluste mussten die Tariflohnbeschäftigten mit minus 1,9\% in Portugal hinnehmen, gefolgt von Italien mit minus 1,8 \% und den Niederlanden mit minus 1,4 \%. Die mit Abstand stärksten Reallohnzuwächse gab es bei den Tariflöhnen mit 1,9 \% in Schweden. Positive Reallohnzuwächse konnten außerdem die Tariflohnbeschäftigten in Österreich (0,7 \%), Deutschland $(0,6 \%)$ und Belgien $(0,4 \%)$ verzeichnen.

Im Jahr 2013 wirken sich die niedrigeren Inflationsraten in einigen Ländern positiv auf die Reallohnzuwächse bei den Tariflöhnen aus. Spitzenreiter ist auch 2013 Schweden mit einem erneuten Reallohnplus von $1,9 \%$, gefolgt nun von Deutschland mit einem Plus von 1,1\%. Die stärksten Reallohnverluste deuten sich hingegen mit jeweils minus $1,7 \%$ in Spanien und den Niederlanden an.

\section{Die Entwicklung der Effektivlöhne}

Bei der Analyse der Effektivlöhne wird im Folgenden erneut auf die AMECO-Datenbank der Europäischen Kommission zurückgegriffen, deren Ursprungsdaten aus den nationalen volkswirtschaftlichen Gesamtrechnungen stammen. ${ }^{5}$ Im Jahr 2012 stiegen danach die effektiven Nominallöhne im EU-Durchschnitt um 1,9 \%. Gegenüber 2,3 \% im Jahr 2010 und 2,2 \% im Jahr 2011 zeigt die Nominallohnentwicklung damit eine leicht rückläufige Tendenz, die sich jedoch 2013 bei prognostizierten 2,0\% nicht fortsetzen dürfte (Tabelle 3).

Hinter den EU-Durchschnittsdaten verbergen sich allerdings erhebliche nationale Unterschiede. In insgesamt zehn EU-Staaten lagen die Nominallohnzuwächse im Jahr 2012 bei $3 \%$ und mehr. Darunter befanden sich mehrheitlich Staaten aus Osteuropa sowie Belgien, Finnland und Österreich. Den höchsten Nominallohnanstieg gab es mit 6,7 \% in Estland. In den meisten westeuropäischen Ländern lagen die Nominallohnzuwächse hingegen zwischen 1 und $2 \%$. Schließlich gab es 2012 vier Länder, in denen die Beschäf-
ABB. 3

\section{Reale Entwicklung der Tariflöhne 2012 - 2013*}

Veränderungen in Prozent zum Vorjahr

$2012=2013$

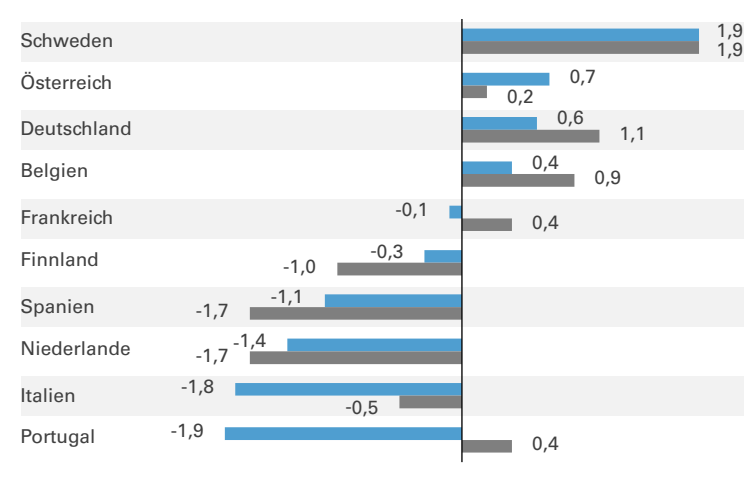

* deflationiert um den Harmonisierten Verbraucherpreisindex (HVPI). 2013: vorläufige Daten auf der Grundlage des ersten Halbjahres.

Quelle: Nationale Angaben, Berechnungen des WSI.

Mitteilungen

tigten mit Nominallohnverlusten konfrontiert waren. Am stärksten gingen die Nominallöhne mit minus 4,2 \% in Griechenland zurück, gefolgt von Portugal mit minus $2,7 \%$ sowie Slowenien und Spanien mit minus $0,4 \%$ bzw. minus $0,3 \%$.

Für das Jahr 2013 erwartet die Europäische Kommission insgesamt eine sehr differenzierte Entwicklung. Extrem hohe Nominallohnverluste werden mit minus 7 \% für Griechenland und minus 7,5 \% für Zypern prognostiziert. Demgegenüber wird für Spanien mit $1,4 \%$ und Portugal mit $3,1 \%$ wieder mit Nominallohnzuwächsen gerechnet. Stagnierende oder leicht rückläufige Nominallöhne soll es in Irland, Slowenien und Ungarn geben. Die meisten westeuropäischen Länder weisen demgegenüber Nominallohnsteigerungen zwischen 1,8\% und 2,6\% auf. Oberhalb von $3 \%$ werden die Zuwächse in Finnland und Schweden sowie wiederum in einer Reihe osteuropäischer Länder liegen, darunter vor allem die baltischen Staaten sowie Bulgarien und Rumänien. Dabei ist zu berücksichtigen, dass die vergleichsweise hohen Lohnsteigerungen in vielen Ländern Osteuropas auch auf Basiseffekten beruhen, die auf der Grundlage eines insgesamt immer noch sehr niedrigen Lohnniveaus zustande kommen.

Vor dem Hintergrund vergleichsweise hoher Inflationsraten blieben im Jahr 2012 die Nominallöhne zumeist

5 Streng genommen werden in der AMECO-Datenbank der Europäischen Kommission keine "Löhne", sondern "Arbeitnehmerentgelte“ (definiert als Bruttolöhne plus Arbeitgeberanteile zur Sozialversicherung) ausgewiesen. Der hier im Folgenden verwendete weite Lohnbegriff umfasst demnach die gesamten Arbeitskosten. 
TABELLE 3

Lohnentwicklung und Verteilungsbilanz in der Europäischen Union 2010-2013*

Angaben in Prozent

\begin{tabular}{|c|c|c|c|c|c|c|c|c|c|c|c|c|}
\hline & \multicolumn{4}{|c|}{ Nominallöhne } & \multicolumn{4}{|c|}{ Reallöhne } & \multicolumn{4}{|c|}{ Verteilungsbilanz } \\
\hline & 2010 & 2011 & 2012 & 2013 & 2010 & 2011 & 2012 & 2013 & 2010 & 2011 & 2012 & 2013 \\
\hline \multicolumn{13}{|l|}{ Nordeuropa } \\
\hline Dänemark & 2,6 & 1,6 & 1,2 & 1,4 & 0,5 & $-1,1$ & $-1,2$ & 0,2 & $-3,5$ & $-2,6$ & $-1,1$ & $-0,7$ \\
\hline Finnland & 1,8 & 3,4 & 3,0 & 3,3 & 0,1 & 0,1 & $-0,1$ & 0,8 & $-3,3$ & $-1,5$ & 0,4 & 0,2 \\
\hline Schweden & 3,1 & 0,8 & 2,8 & 3,1 & 1,2 & $-0,6$ & 1,8 & 2,2 & $-4,3$ & $-1,9$ & 1,7 & 0,9 \\
\hline \multicolumn{13}{|l|}{ Westeuropa } \\
\hline Belgien & 1,4 & 3,1 & 3,3 & 2,4 & $-1,0$ & $-0,3$ & 0,7 & 1,0 & $-2,7$ & $-0,7$ & 1,0 & 1,1 \\
\hline Deutschland & 2,4 & 3,0 & 2,5 & 2,4 & 1,2 & 0,5 & 0,3 & 0,6 & $-2,3$ & $-1,1$ & 0,8 & 0,8 \\
\hline Frankreich & 2,6 & 2,8 & 1,9 & 1,3 & 0,8 & 0,5 & $-0,4$ & 0,1 & $-1,1$ & $-0,7$ & $-0,5$ & 0,2 \\
\hline Großbritannien & 2,8 & 1,8 & 2,3 & 2,6 & $-0,5$ & $-2,6$ & $-0,6$ & $-0,2$ & $-2,1$ & $-3,1$ & 0,4 & $-0,2$ \\
\hline Irland & $-3,2$ & $-0,2$ & 1,8 & 0,3 & $-1,7$ & $-1,4$ & $-0,2$ & $-1,0$ & $-5,1$ & $-4,6$ & $-1,7$ & $-1,9$ \\
\hline Luxemburg & 2,7 & 2,0 & 1,2 & 2,2 & $-0,1$ & $-1,7$ & $-1,7$ & 0,4 & $-1,2$ & $-0,5$ & 0,3 & 1,2 \\
\hline Niederlande & 1,5 & 1,8 & 1,3 & 1,8 & 0,5 & $-0,7$ & $-1,6$ & $-1,1$ & $-1,7$ & $-1,2$ & $-1,5$ & $-1,3$ \\
\hline Österreich & 1,3 & 2,3 & 3,0 & 2,1 & $-0,4$ & $-1,2$ & 0,4 & 0,1 & $-1,9$ & $-2,5$ & 0,8 & 0,2 \\
\hline \multicolumn{13}{|l|}{ Südeuropa } \\
\hline Griechenland & $-2,6$ & $-3,4$ & $-4,2$ & $-7,0$ & $-7,3$ & $-6,5$ & $-5,2$ & $-6,2$ & $-4,8$ & $-4,9$ & $-7,4$ & $-5,5$ \\
\hline Italien & 2,8 & 1,3 & 1,0 & 1,2 & 1,1 & $-1,6$ & $-2,3$ & $-0,4$ & $-1,7$ & $-1,9$ & $-1,0$ & $-0,1$ \\
\hline Malta & 1,0 & 0,5 & 2,4 & 1,8 & $-1,1$ & $-2,0$ & $-0,8$ & $-0,1$ & $-2,2$ & $-1,0$ & 0,4 & 0,3 \\
\hline Portugal & 2,0 & $-0,7$ & $-2,7$ & 3,1 & 0,6 & $-4,2$ & $-5,5$ & 2,4 & $-2,9$ & $-4,2$ & $-6,6$ & 0,8 \\
\hline Spanien & 0,0 & 0,7 & $-0,3$ & 1,4 & $-2,0$ & $-2,4$ & $-2,7$ & $-0,1$ & $-4,0$ & $-4,5$ & $-5,9$ & $-2,1$ \\
\hline Zypern & 2,6 & 3,3 & 1,6 & $-7,5$ & $-0,0$ & $-0,2$ & $-1,5$ & $-8,5$ & $-1,5$ & $-0,3$ & $-3,2$ & $-6,3$ \\
\hline \multicolumn{13}{|l|}{ Osteuropa } \\
\hline Bulgarien & 11,2 & 8,6 & 5,6 & 4,4 & 8,2 & 5,2 & 3,2 & 2,3 & 2,8 & $-0,2$ & $-2,2$ & $-0,7$ \\
\hline Estland & 1,8 & $-0,2$ & 6,7 & 5,7 & $-0,9$ & $-5,3$ & 2,4 & 2,2 & $-9,4$ & $-6,5$ & 1,5 & $-0,5$ \\
\hline Kroatien & 1,9 & 3,0 & 3,2 & 4,2 & 0,8 & 0,8 & $-0,1$ & 1,1 & $-2,2$ & $-1,6$ & $-2,1$ & $-2,2$ \\
\hline Lettland & $-6,7$ & 17,2 & 3,9 & 3,1 & $-5,5$ & 13,0 & 1,6 & 1,8 & $-9,6$ & $-1,9$ & $-1,3$ & $-0,2$ \\
\hline Litauen & $-0,4$ & 3,6 & 2,4 & 4,1 & $-1,6$ & $-0,5$ & $-0,7$ & 2,0 & $-8,6$ & $-4,3$ & $-2,6$ & 0,3 \\
\hline Polen & 4,7 & 4,0 & 3,1 & 2,6 & 2,1 & 0,2 & $-0,6$ & 1,2 & $-1,3$ & $-3,3$ & $-2,2$ & $-0,3$ \\
\hline Rumänien & $-3,3$ & 4,2 & 5,2 & 5,9 & $-9,4$ & $-1,7$ & 1,8 & 1,6 & $-8,5$ & $-4,9$ & 3,0 & 0,4 \\
\hline Slowakei & 5,1 & 1,1 & 2,0 & 1,7 & 4,4 & $-3,0$ & $-1,7$ & $-0,3$ & $-1,6$ & $-4,4$ & $-3,7$ & $-1,2$ \\
\hline Slowenien & 3,9 & 1,6 & $-0,4$ & 0,1 & 1,8 & $-0,5$ & $-3,2$ & $-2,1$ & $-1,7$ & $-2,7$ & $-2,1$ & $-1,8$ \\
\hline Tschechien & 3,6 & 2,7 & 1,8 & 1,5 & 2,3 & 0,6 & $-1,7$ & $-0,4$ & $-1,2$ & $-1,0$ & $-0,0$ & $-0,0$ \\
\hline Ungarn & $-0,3$ & 3,0 & 4,6 & $-0,4$ & $-5,0$ & $-0,9$ & $-1,0$ & $-3,0$ & $-5,6$ & $-2,1$ & 0,7 & $-3,2$ \\
\hline EU 28 & 2,3 & 2,2 & 1,9 & 2,0 & 0,2 & $-0,9$ & $-0,7$ & 0,2 & $-2,4$ & $-2,2$ & $-0,7$ & $-0,1$ \\
\hline
\end{tabular}

*Nominallöhne $=$ Nominale Arbeitnehmerentgelte pro Arbeitnehmer, Veränderung in \% gegenüber dem Vorjahr

Reallöhne = Nominale Arbeitnehmerentgelte pro Arbeitnehmer deflationiert um die Entwicklung des Harmonisierten Verbraucherpreisindex, Veränderung in \% gegenüber dem Vorjahr.

Verteilungsbilanz $=$ Saldo des jährlichen Nominallohnzuwachses und des Verteilungsspielraums (vgl. Tabelle 2) in Prozentpunkten.

Angaben für 2013: Prognose der Europäischen Kommission.

Quelle: AMECO Datenbank der Europäischen Kommission, Version: 3.5.2013, Berechnungen des WSI.

hinter den Preisen zurück, sodass die effektiven Reallöhne einen negativen Entwicklungstrend aufweisen. Insgesamt mussten die Beschäftigten in 20 von 28 EU-Staaten Reallohnverluste hinnehmen. Im EU-Durchschnitt ging das Reallohnniveau um 0,7 \% zurück, nachdem es im Vorjahr bereits um $0,9 \%$ gesunken war (Tabelle 3 ). Von den acht Ländern mit Reallohnzuwächsen kamen vier aus Osteuropa, drei aus Westeuropa (darunter Deutschland mit einem leichten Reallohnplus von 0,3\%), eins aus Nordeuropa und keins aus Südeuropa. Im Jahr 2013 wird sich nach Ansicht der Europäischen Kommission die Reallohnentwicklung wieder leicht verbessern und bei einem mageren Plus von $0,2 \%$ im EU-Durchschnitt stagnieren. Allerdings wird nach wie vor für zwölf EU-Staaten ein Reallohnrückgang erwartet. Und in Ländern mit Reallohnzuwächsen fallen diese zumeist eher bescheiden aus.

Angesichts sinkender Reallöhne ist es nicht weiter verwunderlich, dass auch die Verteilungsbilanz, die sich aus 


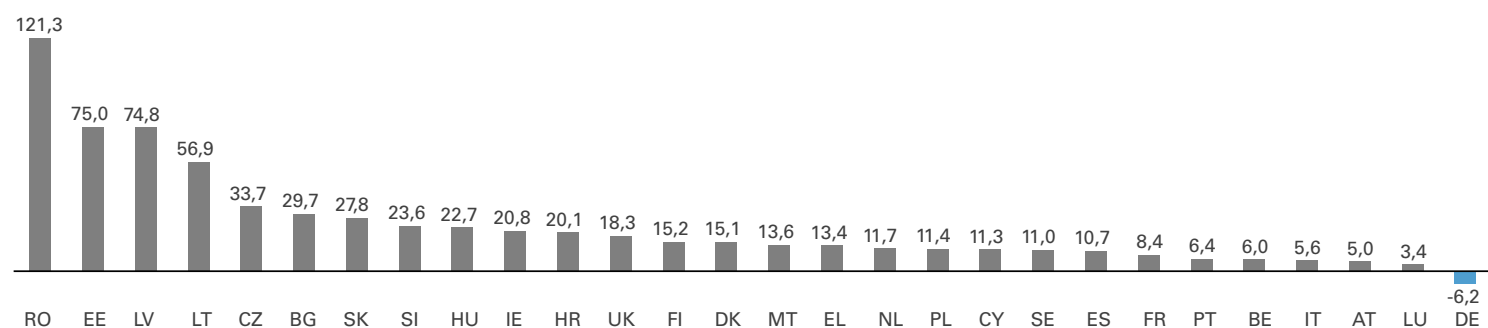

$\mathrm{AT}=$ Österreich, $\mathrm{BE}=$ Belgien, $\mathrm{BG}=$ Bulgarien, $\mathrm{CY}=$ Zypern, $\mathrm{CZ}=$ Tschechische Republik, DE $=$ Deutschland, $\mathrm{DK}=$ Dänemark, $\mathrm{EE}=\mathrm{Estland}, \mathrm{EL}=\mathrm{Griechenland}$, $\mathrm{ES}=$ Spanien, $\mathrm{FI}=$ Finnland, $\mathrm{FR}=$ Frankreich, $\mathrm{HR}=$ Kroatien, $\mathrm{HU}=$ Ungarn, $\mathrm{IE}=$ Irland, IT = Italien, $\mathrm{LT}=\mathrm{Litauen}, \mathrm{LU}=\mathrm{Luxemburg}, \mathrm{LV}=\mathrm{Lettl}$ land, MT $=\mathrm{Malta}$ $\mathrm{NL}=$ Niederlande, $\mathrm{PL}=$ Polen, $\mathrm{PT}=$ Portugal, RO = Rumänien, $\mathrm{SE}=$ Schweden, $\mathrm{SI}$ = Slowenien, $\mathrm{SK}=$ Slowakei, UK = Großbritannien

*deflationiert um den Harmonisierten Verbraucherpreisindex (HVPI).

Quelle: Ameco-Datenbank; Berechnungen des WSI.

dem Saldo aus Nominallohnentwicklung und dem Verteilungsspielraum ergibt, 2012 in vielen europäischen Ländern wieder negativ ist. Aufgrund sinkender Verteilungsspielräume infolge einer rückläufigen Preis- und Produktivitätsentwicklung hat sich die Verteilungsbilanz gegenüber den Vorjahren jedoch relativ gesehen etwas verbessert. Im EU-Durchschnitt lag die Verteilungsbilanz 2012 0,7 Prozentpunkte unterhalb des Verteilungsspielraums gegenüber minus $2,2 \%$ im Jahr 2011. Für 2013 erwartet die Europäische Kommission mit minus 0,1 Prozentpunkten sogar eine beinahe ausgeglichene Verteilungsbilanz. Nachdem in den Jahren 2010 und 2011 fast ausnahmslos alle EU-Staaten eine negative Verteilungsbilanz aufwiesen, liegen 2012 und 2013 in einigen Ländern die Lohnzuwächse erstmals wieder leicht oberhalb des Verteilungsspielraums. Dies gilt mit einer positiven Verteilungsbilanz von jeweils 0,8 Prozentpunkten in den Jahren 2012 und 2013 auch für Deutschland, wo die Verteilungsbilanz in den letzten beiden Jahrzehnten (mit Ausnahme einiger weniger Krisenjahre) ansonsten durchweg negativ war. Allerdings sind die Verschiebungen in der Verteilungsbilanz primär konjunktureller Natur und weniger das Ergebnis einer expansiven Lohnpolitik.

\section{Ausblick: Schwache Lohnentwicklung verstärkt ökonomische Stagnations- tendenzen}

Die Lohnentwicklung der Jahre 2012 und 2013 zeigt im Vergleich zu den Vorjahren kaum Unterschiede und fügt sich damit nahtlos in das neue lohnpolitische Entwicklungsmuster ein, das seit der Weltwirtschaftskrise 2009ff. in
Europa prägend ist. Bis zum Ausbruch der Krise war die Lohnentwicklung durch mehr oder weniger hohe Reallohnzuwächse geprägt (Abbildung 4). Im Zeitraum 2001 bis 2009 zeigten insbesondere die osteuropäischen Länder sehr hohe Reallohnsteigerungen und unterstrichen damit ihren ökonomischen Aufholprozess gegenüber den übrigen EU-Staaten. Außerhalb Osteuropas kam es in so unterschiedlichen Staaten wie Irland und Großbritannien, Griechenland sowie den nordeuropäischen Ländern Dänemark und Finnland zu relativ hohen Reallohnsteigerungen. Zu der Gruppe mit vergleichsweise niedrigen Reallohnzuwächsen gehörten dagegen vor allem die deutschen Nachbarstaaten Frankreich, Belgien, Luxemburg und Österreich, aber auch die heute im Krisenfokus stehenden südeuropäischen Staaten Italien, Portugal und Spanien. Eine absolute lohnpolitische Sonderrolle wurde schließlich von Deutschland eingenommen, das während der letzten Dekade als einziges Land in Europa erhebliche Reallohnrückgänge zu verzeichnen hatte (Schulten 2011).

Seit 2010 zeigt die Lohnentwicklung in Europa ein vollkommen neues Muster, in dem Reallohnverluste zum dominierenden Trend werden. Unter Einbeziehung der Prognosedaten der Europäischen Kommission ist die Reallohnentwicklung im Zeitraum 2010 bis 2013 in 18 von 28 EU-Staaten rückläufig (Abbildung 5). Besonders extrem sind die Reallohnverluste mit fast $23 \%$ in Griechenland, gefolgt von Ungarn mit fast $15 \%$ und Zypern mit $10 \%$. Hohe Reallohnrückgänge mit Werten zwischen $6,5 \%$ und $8 \%$ verzeichnen darüber hinaus auch Portugal, Spanien und Rumänien. Auf der anderen Seite existieren mit Bulgarien, Lettland und Schweden lediglich drei Staaten, in denen es im selben Zeitraum größere Reallohnzuwächse gab. Auch Deutschland konnte erstmals seit Langem wieder eine positive Reallohnbilanz aufweisen, wobei hierdurch jedoch bislang nur knapp die Hälfte der Reallohnverluste der 2000er Jahre ausgeglichen werden konnte. 


\section{Entwicklung der Reallöhne in der EU 2010 - 2013}

Angaben in Prozent*

20,1

\begin{tabular}{lllllllll}
10,4 & & & & & & & & \\
& 4,8 & 2,8 & 2,8 & 2,5 & 1,1 & 0,9 & 0,8 & 0,5 \\
\hline & & & &
\end{tabular}

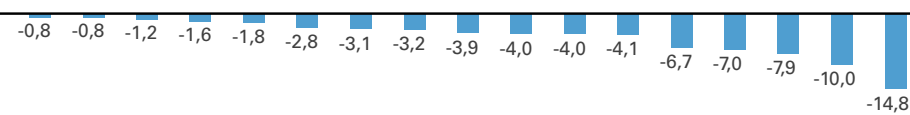

BG

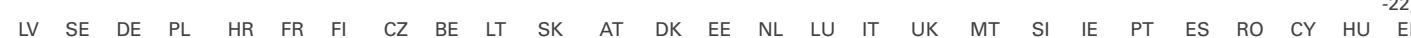

$\mathrm{AT}=$ Österreich, $\mathrm{BE}=$ Belgien, $\mathrm{BG}=$ Bulgarien, $\mathrm{CY}=$ Zypern, $\mathrm{CZ}=$ Tschechische Republik, DE $=$ Deutschland, $\mathrm{DK}=\mathrm{Dänemark}, \mathrm{EE}=\mathrm{Estland}, \mathrm{EL}=\mathrm{Griechenland}$ $\mathrm{ES}=$ Spanien, $\mathrm{FI}=$ Finnland, $\mathrm{FR}=$ Frankreich, $\mathrm{HR}=$ Kroatien, $\mathrm{HU}=$ Ungarn, IE = Irland, IT = Italien, LT = Litauen, LU = Luxemburg, LV = Lettland, MT = Malta $\mathrm{NL}=$ Niederlande, $\mathrm{PL}=$ Polen, SPT = Portugal, RO = Rumänien, SE = Schweden, SI = Slowenien, SK = Slowakei, UK = Großbritannien

*deflationiert um den Harmonisierten Verbraucherpreisindex (HVPI).

Daten für 2013: Frühjahrsprognose der Europäischen Kommission.

Quelle: Ameco-Datenbank; Berechnungen des WSI.

Das durch Reallohnverluste geprägte neue lohnpolitische Entwicklungsmuster in Europa ist nicht nur Ausdruck einer durch die Wirtschaftskrise und hohe Arbeitslosigkeit strukturell geschwächten Verhandlungsposition der Gewerkschaften, sondern auch das Ergebnis eines neuen lohnpolitischen Interventionismus (Schulten/Müller 2013). So hat sich im Zuge der Krise innerhalb der EU ein neues System der Economic Governance herausgebildet, das auf eine festere Koordinierung der europäischen Wirtschaftspolitik zielt. Im Rahmen des sogenannten „Europäischen Semesters“, das einen jährlich wiederkehrenden Koordinierungszyklus darstellt, werden regelmäßig die Lohnentwicklungen in allen EU-Staaten überprüft und Empfehlungen für die nationale Lohnpolitik ausgesprochen. Letztere beziehen sich nicht nur auf die Höhe einer „angemessenen“ Entwicklung der Löhne und Lohnstückkosten, sondern auch auf eine „nach unten gerichtete Flexibilität“ der Tarifvertragssysteme.

Im Ergebnis dieser neuen europäischen Economic Governence kommt es zu massiven staatlichen Interventionen in die Lohnpolitik, die vor allem Lohnkürzungen und Lohnstopps im öffentlichen Sektor (Europäische Kommission 2013b) und bei den gesetzlichen Mindestlöhnen (Schulten 2013) umfassen. Außerdem haben viele europäische Länder einen aktiven Umbau der Tarifvertragssysteme begonnen, der einer Strategie der radikalen Dezentralisierung folgt und mitunter bereits zu einer weitreichenden Zerstörung von Flächentarifvertragsstrukturen geführt hat (Schulten/Müller 2013). Am weitesten ausgeprägt ist diese Entwicklung derzeit in Ländern wie z. B. Griechenland, Portugal oder Irland, die unter dem direkten Einfluss der sogenannten „Troika“ aus Europäischer Kommission, Europäischer Zentralbank (EZB) und dem Internationalen Währungsfond (IWF) stehen.
Aus Sicht der vorherrschenden Krisenpolitik in Europa stellen diese lohnpolischen Eingriffe notwendige „Strukturreformen“ dar, die in den betroffenen Ländern helfen sollen, wettbewerbsfähiger zu werden und ihre Leistungsbilanzdefizite abzubauen. In diesem Sinne interpretiert z. B. die Europäische Kommission (2013a) die in vielen Ländern zu beobachtenden Reallohneinschnitte als „Erfolg" dieser Politik und prognostiziert für die kommenden Jahre eine spürbare konjunkturelle Erholung.

Bislang hat die Europäische Kommission allerdings ihre seit Ausbruch der Krise 2009ff. stets zu optimistischen Prognosen immer wieder nach unten korrigieren müssen (Marterbauer 2013). Dies deutet darauf hin, dass die derzeit vorherrschende Krisenpolitik in Europa die ökonomische Lage keineswegs verbessert, sondern im Gegenteil weiter verschärft hat. Letzteres wurde mittlerweile sogar durch eine viel beachtete Studie des IWF bestätigt, in der offen eingestanden wurde, dass die negativen Einflüsse der Austeritätspolitik auf die Entwicklung der gesamtwirtschaftlichen Nachfrage systematisch unterschätzt wurden (Blanchard/ Leigh 2013). Obwohl sich offensichtlich ein ähnlicher $\mathrm{Zu}$ sammenhang auch für die anhaltend schwache Lohnentwicklung in Europa konstatieren lässt, fordert der gleiche IWF nach wie vor die Fortsetzung neoliberaler Strukturreformen auf dem Arbeitsmarkt (Blanchard et al. 2013). Im Falle von Spanien hat der IWF beispielsweise erst unlängst den dortigen Arbeitgeberverbänden und Gewerkschaften angeraten, im Rahmen eines nationalen Sozialpaktes eine zusätzliche landesweite (Nominal)Lohnkürzung von $10 \%$ zu vereinbaren (IWF 2013, S.14).

Es ist daher zu befürchten, dass der internationale Druck auf die Lohnpolitik in vielen europäischen Ländern weiter anhalten wird und die Lohnentwicklung in der gesamten EU ihren deflationären Kurs weiter fortsetzt. Umso wichtiger ist es, dass Deutschland und einige andere west- und 
nordeuropäische Länder mit positiver Leistungsbilanz dem entgegentreten und - auch mithilfe einer in den nächsten Jahren deutlich expansiveren Lohnentwicklung - die wirtschaftliche Dynamik in ganz Europa fördern. Schließlich ist es gerade Deutschland, das derzeit in vielen europäischen Ländern als Vorbild für neoliberale Arbeitsmarktreformen und eine Politik der Lohnkürzung gepriesen wird. Insofern ist es auch für Europa heute von größter Bedeutung, dass Deutschland wieder einen anderen Weg geht, der auf eine erneute Re-Regulierung des Arbeitmarktes und eine deutlich stärkere Lohndynamik setzt.

\section{LITERATUR}

Aumayr-Pintar, C./Fric, K. (2013): Developments in collectively agreed pay 2012: Eurofound, Dublin

Bispinck, R./Schulten, T. (2012): Collectively agreed wages in Germany, Country-Study for the CAWIE-Project, Leuven, https://hiva.kuleuven.be/resources/ docs/vorming/20121129_CAWIEpaper_Germany.pdf

Blanchard, O./Jaumotte, F./Loungani, P. (2013): Labour Market Policies and IMF Advice in Advanced Economies During the Great Recession, IMF Staff Discussion Note SDN/13/02, Washington, D.C.

Blanchard, O./Leigh, D. (2013): Growth Forecast Errors and Fiscal Multipliers, IMF Working Paper WP/13/1, Washington, D.C.

Europäische Kommission (2013a): European Economic Forecast - Spring 2013 European Economy 2/2013, http://ec.europa.eu/economy_finance/publications/ european_economy/2013/pdf/ee2_en.pdf

Europäische Kommission (2013b): Industrial Relations in Europe 2012, Luxemburg

Herr, H./Horn, G. A. (2012): Lohnpolitik heute, IMK Policy Brief, Mai, Düsseldorf Horn, G./Herzog-Stein, A./Hohlfeld, P./Lindner, F./Rannenberg, A./Rietzler, K./ Stephan, S./Tober, S./Zwiener, R. (2013): Krise überwunden? Prognose der wirtschaftlichen Entwicklung 2013/2014, IMK-Report (86), Düsseldorf, Oktober Internationaler Währungsfond (IWF) (2013): Spain, IMF Country Report 13/244, http://www.imf.org/external/pubs/ft/scr/2013/cr13244.pdf
Marterbauer, M. (2013): Die neoklassische Wirtschaftspolitik der EU ist gescheitert: Kammer für Arbeiter und Angestellte für Wien, in: Infobrief EU \& International (3), S. 1-6

Schubert, A. (2012): Experimental data as part of the ECB's statistical production and dissemination policy, Paper, präsentiert auf der European Conference on Quality in Official Statistics, 29. Mai - 1. Juni in Athen, http://www.q2012.gr/articlefiles/sessions/26.2-Experimental-statisticsECBAurel-Schubert.pdf

Schulten, T. (2011): Deutschlands lohnpolitische Sonderrolle in Europa, in: Bispinck, R. (Hrsg.): Zwischen Beschäftigungswunder und Lohndumping? Tarifpolitik in und nach der Krise, Hamburg, S. 47-58

Schulten, T. (2013): WSI-Mindestlohnbericht 2013 - Anhaltend schwache Mindestlohnentwicklung in Europa, in: WSI-Mitteilungen 66 (2), S. 126-132, http://www.boeckler.de/wsimit_2013_02_schulten.pdf

Schulten, T./Müller, T. (2013): Ein neuer europäischer Interventionismus? Die Auswirkungen des neuen Systems der europäischen Economic Governance auf Löhne und Tarifpolitik, in: Wirtschaft und Gesellschaft 39 (3), S. 291-321 Van Gyes, G. (2012): Indicators of collectively-agreed wages in the Eurozone. A quality report: HIVA K.U. Leuven, Policy Paper CAWIE Project, November, https://hiva.kuleuven.be/resources/pdf/publicaties/R1495_CAWIE_Paper1_ GVanGyes.pdf

Visser, J. (2013): Wage Bargaining Institutions - from crisis to crisis, DG Ecfin Economic Papers (488), April, Brüssel

\section{AUTOR}

THORSTEN SCHULTEN, Dr., Wissenschaftler im Wirtschafts- und Sozialwissenschaftlichen Institut (WSI) in der Hans-Böckler-Stiftung. Arbeitsschwerpunkte: Arbeits- und Tarifpolitik in Europa.

thorsten-schulten@boeckler.de 